principal causes of the condition, it seems illogical to use local greasy applications or haemorrhoidectomy as the mainstay of treatment. Yet throughout the world, until recent years, classical medicine has espoused these methods. Many of the enlightened now regard both treatments as refuges of the proctologically destitute. They still have a place, but only within the whole armament of stool-bulking agents, anal dilatation, sphincterotomy, and mucosal fixation by fire, oil, or frost.

J AlEXANDER-WILliamS

Consultant Surgeon,

General Hospital,

Birmingham B4 6NH

1 Thomson WHF. The nature of haemorrhoids. Br $\mathcal{F}$ Surg 1975;62:542-52.

2 Lord PH. Conservative management of haemorrhoids : 2-dilatation treatment. Clin Gastroenterol 1975;4:401-8.

\section{Does control of risk factors prevent coronary heart disease?}

The seven-year American Multiple Risk Factor Intervention Trial has ended with inconclusive results-and leaving many questions unanswered. ${ }^{1}$ Whether intervention to control cigarette smoking, hypertension, and hypercholesterolaemia alters mortality from coronary heart disease in men at high risk remains uncertain. And an appalling consequence of these results is that the central question is likely to remain unresolved indefinitely, for this vast trial, costing $\$ 115$ million and based on 28 institutions with 250 investigators, is unlikely ever to be repeated.

The trial population was 12866 men, selected from 361662 men who volunteered for health screening through their employment. The 12866 represented the upper $15 \%$ of a risk score distribution (two-thirds were in the upper 10\%) based on smoking, blood pressure, and plasma cholesterol concentration. None had clinical or electrocardiographic evidence of coronary heart disease.

Half the selected group was randomly allocated to an intensive intervention programme conducted by behavioural scientists, nutritionists, nurses, physicians, and health counsellors; they were seen every four months or more frequently to ensure adequate control of the risk factors. The other half received a medical examination once yearly and no specific advice about control of risk factors, other than that given by their doctors. Randomisation of characteristics associated with coronary heart disease was excellent and established that the two groups were comparable on admission to the trial. Less than $10 \%$ of each group was lost to follow-up over the first six years.

In the intervention group simultaneous intervention programmes were mounted to stop cigarette smoking, to control raised blood pressure (mostly with thiazides), and to alter the diet to reduce hypercholesterolaemia. Initially, the aim of the dietary changes was to reduce the intake of saturated fat to less than $10 \%$ of energy and the daily intake of cholesterol to less than $300 \mathrm{mg}$; later these limits were narrowed to less than $8 \%$ of energy and $250 \mathrm{mg}$ of cholesterol.

After six years half of the men in the intervention group had stopped smoking ( $43 \%$ at one year), the mean diastolic blood pressure had fallen from $91 \mathrm{~mm} \mathrm{Hg}$ to $81 \mathrm{~mm} \mathrm{Hg}$ (and by $12 \%$ in those initially with $95 \mathrm{~mm} \mathrm{Hg}$ or more), and the average plasma cholesterol concentration was reduced from $6.2 \mathrm{mmol} / 1$ $(240 \mathrm{mg} / 100 \mathrm{ml})$ by $0.3 \mathrm{mmol} / 1(12 \mathrm{mg} / 100 \mathrm{ml})-5 \%$. Substantial decreases also occurred in all risk factors in the nonintervention group, but statistically significant differences were achieved between the intervention and non-intervention groups in each of the three major risk factors at each annual visit. The difference in cigarette smoking between the groups exceeded the goal of the trial. The difference in diastolic blood pressure was, however, only three-quarters of the design goal, and for cholesterol only half of the goal-a difference of no more than $0.1 \mathrm{mmol} / 1(4.6 \mathrm{mg} / 100 \mathrm{ml})$.

An analysis based on averaging calculated coronary heart disease risk for each participant over the six years of follow-up indicated a potential net reduction of mortality of $22 \%$ (compared with a projected $27 \%$ ) between the groups as a result of the difference in reduction of risk factors, but no significant reduction in mortality was actually achieved.

What, then, went wrong? The investigators had not expected any decreases in risk factors in the non-intervention group; it was a major miscalculation that the design of the Multiple Risk Factor Intervention Trial did not allow for any change in blood pressure or cholesterol concentrations in this group. The investigators seriously underestimated the effects on high-risk men of knowledge of that risk, of the influence of those in the intervention group on those in the non-intervention group in the same industry or office, of annual reviews in the framework of the trial, and of providing doctors with annual reports and data on their patients' progress in the trial. The non-intervention group also had a lower-than-expected mortality, reducing the chance of detecting a significant difference between the groups. Indeed, the non-intervention group was not a satisfactory control group and this could have been predicted at the outset.

The trial report has some worrying features. Several subgroups have been analysed outside the randomised controlled design; this gives grounds for concern because of the small residual numbers in such subgroups, because some were defined post hoc, and because other confounding factors cannot be controlled. These analyses provide encouragement in only one respect. Non-hypertensive hypercholesterolaemic smokers had a lower $(-49 \%)$ mortality from coronary heart disease in the intervention group than in the non-intervention group, though this difference was not statistically significant. But hypertensive men with initial electrocardiographic abnormalities did worse with higher mortality from coronary heart disease $(+65 \%)$ and total mortality $(+58 \%)$. How much of the excess occurred in thiazide-treated men is not clear. After exclusion of the $28 \%$ with abnormal baseline electrocardiograms all subgroups of risk had fewer deaths from coronary heart disease in the intervention group than the nonintervention group: but even this focusing on the healthiest subsections did not provide any significant differences.

The tenor of the report clearly shows that the investigators were very disappointed with the results. Indeed, a degree of rationalisation is evident in the commentary. Three explanations are offered: firstly, that overall intervention to reduce multiple risk factors in high-risk men does not affect coronary heart disease mortality over seven years; secondly, that it does but concurrent changes in the control group prevented its demonstration; or, thirdly, that some aspects of intensive intervention had a deleterious effect in certain subgroups obscuring a truly beneficial effect in others. The authors favour the last explanation but it seems unlikely that the small numbers with such an adverse response, less than a tenth of the 
total study, could have diluted any important positive overall effect.

Is not the second explanation the most likely? The results of this trial are disconcertingly similar to those of a Finnish multifactor intervention trial of primary prevention of coronary heart disease, ${ }^{2}$ though the design of the Finnish study was different in that it comprised two cross-sectional surveys of population samples not selected for high risk. An overall mean net reduction of $17 \%$ in men and of $12 \%$ in women occurred five years later with regard to cigarette smoking, blood pressure, and plasma cholesterol concentrations in the intervention community (North Karelia) compared with the control community (Kuopio). But there was no reduction in morbidity from coronary heart disease. Again, risk factors had been reduced in the control community, too.

Or is the first explanation the correct one ? The incubation period of coronary atherosclerosis is far longer than the sevenyear duration of the trial. ${ }^{3}$ We do not yet know the extent, if any, of regression of coronary lesions which occurs in middleaged men even with such successful control of risk factors as was achieved in this trial. It does not follow that intervention to produce minor regression or non-progression of lesions will lead to fewer clinical manifestations of coronary heart disease in those who already have moderate or advanced obstructive arterial disease. Perhaps cigarette smoking, hypertension, and hypercholesterolaemia are not the relevant risk factors to control at this stage; perhaps prevention of the factors which trigger the onset of the clinical syndromes of coronary heart disease would yield more. Thrombosis is an obvious area for more research in relation to these syndromes, but we are only just beginning to identify which indices have predictive power, ${ }^{4}$ let alone how to intervene for primary prevention.

What positive conclusions can be extracted from the Multiple Risk Factor Intervention Trial ? Stopping cigarette smoking in both the intervention and non-intervention groups appears to have reduced coronary heart disease mortality significantly, though confounding factors cannot be excluded. The results of intervention in the subgroup of smokers and men with hypercholesterolaemia were favourable, supporting the findings of the smaller Oslo study, ${ }^{5}$ which showed a reduction in the incidence of coronary heart disease with cessation of smoking and dietary intervention to lower lipid concentrations in nonhypertensive men in high-risk categories. More cannot be said.

What might be expected from the continuing World Health Organisation European trial in industry? This comprises 63732 men aged 40-59 in 44 pairs of factories in Britain, Belgium, Italy, Poland, and Spain; $17 \%$ of these are categorised as high risk for coronary heart disease. An interim report ${ }^{6}$ on the changes in risk factors after two and four years of intervention makes depressing reading. Changes were smaller than expected and not completely consistent or sustained. The authors comment that, despite an estimated fall of $14 \%$ in coronary heart disease risk in the whole group and of $24 \%$ in the high-risk subgroup after four years, no equivalent fall in incidence of coronary heart disease may be shown even in a study of this size. The British experience was reported earlier ${ }^{7}$ : while showing that coronary risk factors can be changed in a working population at modest cost, they were also small and not sustained.

The indecisive results of the Multiple Risk Factor Intervention Trial will not alter the nihilism of the "abominable no-men,"8 though they should temper the fervour of the evangelists-the twin dangers which threaten the formulation of a responsible policy towards prevention of the principal cause of death in developed countries. Much as we might like to think otherwise, it is not yet possible to prevent coronary heart disease in the community-let alone in an individual. And the falling mortality from coronary heart disease in the United States remains largely unexplained and cannot be ascribed wholly to preventive measures. Nevertheless, sensible pragmatic health education should continue to insist on stopping cigarette smoking-and at least this can be reinforced by the results of the Multiple Risk Factor Intervention Trial. Surely, too, a prudent diet with reduction of fat energy and control of obesity and of sloth are sound policies for improving the public health.

M F OLIVER

Duke of Edinburgh Professor of Cardiology,

Cardiovascular Research Unit

University of Edinburgh,

Edinburgh EH8 9FX

${ }^{1}$ Multiple Risk Factor Intervention Trial. Risk factor changes and mortality results. FAMA 1982;248:1465-77.

2 Puska P, Tuomilehto J, Salonen J. Changes in coronary risk factors during comprehensive five-year community programme to control cardiovascular diseases (North Karelia project). Br Med f 1979;ii:1173-8.

${ }^{3}$ Rose G. Incubation period of coronary heart disease. $\mathrm{Br} \mathrm{Med}$ F $1982 ; 284$ : 1600-1.

4 Meade TW. Risk associations in the thrombotic disorders. Clin Haematol $1981 ; 10$, No 2:391-405.

${ }^{5}$ Hjermann I, Byre KV, Holme I, Leren P. Effect of diet and smoking intervention on the incidence of coronary heart disease. Report from the Oslo Study Group of a Randomised Trial in Healthy Men. Lancet 1981 ;ii:1303-10.

6 World Health Organisation European Collaborative Group. Multifactorial trial in the prevention of coronary heart disease. 2. Risk factor changes at two and four years. Eur Heart $\mathcal{F} 1982 ; 3: 184-90$.

${ }^{7}$ Rose G, Heller RF, Pedoe HT, Christie DGS. Heart disease prevention project: a randomised controlled trial in industry. $\mathrm{Br} \mathrm{Med} \mathcal{F} 1980 ; 280$ : 747-51.

8 Anonymous. Abominable no-men. Times Health Supplement 1982 Feb 26: 10 (col 3-5).

\section{Amaurosis fugax}

Commonly the patient with amaurosis fugax is a man aged over $50^{1}$ who complains of one or more episodes of unilateral loss of vision-“like a curtain coming down." Each episode lasts seconds or minutes, occasionally an hour or more. Examination may suggest that cerebral emboli have occurred in the past $^{12}$ and may disclose a carotid bruit on the same side; the classic, but uncommon, finding is the presence of a glistening cholesterol crystal embolus in a retinal arteriole, often at a bifurcation-the Hollenhorst plaque. Then the diagnosis of amaurosis fugax is plain. Its management is not.

If a plaque is not seen other diagnoses must be considered. When the build-up of symptoms is slower, migraine accompagnée may be considered, even without current or past headache. ${ }^{3}$ Monocular visual loss may also complicate cranial arteritis, Raynaud's disease, papilloedema, and retrobulbar neuritis; it may be associated with arteriolar damage from eclampsia, uraemia, paroxysmal hypertension, and porphyria. ${ }^{4}$ Blood disorders such as polycythaemia, ${ }^{5}$ thrombocytosis, ${ }^{6} 7$ anaemia, and thrombocytopaenic purpura may be considered, as may cardiac lesions-prolapse of the mitral valve, ${ }^{89}$ atrial myxoma, ${ }^{10}$ and bicuspid aortic valve ${ }^{11}$-particularly with episodes lasting longer than an hour. ${ }^{12}$ In practice, however, artery-to-artery embolism accounts for over $90 \%$ of all cases. ${ }^{113}$

A waiting policy is perilous ${ }^{14}$ : about a third of patients with amaurosis fugax may go on to have a stroke within five years. 